\title{
Ultrasound-Accelerated Amide Coupling Reactions Directed toward the Synthesis of 1-Acetyl-3-carboxamide- $\beta$-carboline Derivatives of Biological Importance
}

\author{
N. Sharma ${ }^{a}$ \\ P. Kumaria \\ P. Sharma ${ }^{a}$ \\ N. Bhagat ${ }^{b}$ \\ S. Bhagat*a \\ a Organic Synthesis research Laboratory, Department of Chem- \\ istry, A.R.S.D. College, University of Delhi, New Delhi-110021, \\ India \\ b Instrumentation \& Control Engineering, Netaji Subash Insti- \\ tute of Technology, University of Delhi, Azad Hind Fauz Marg, \\ Dwarka, Delhi-110078, India \\ sunitabhagat28@gmail.com
}

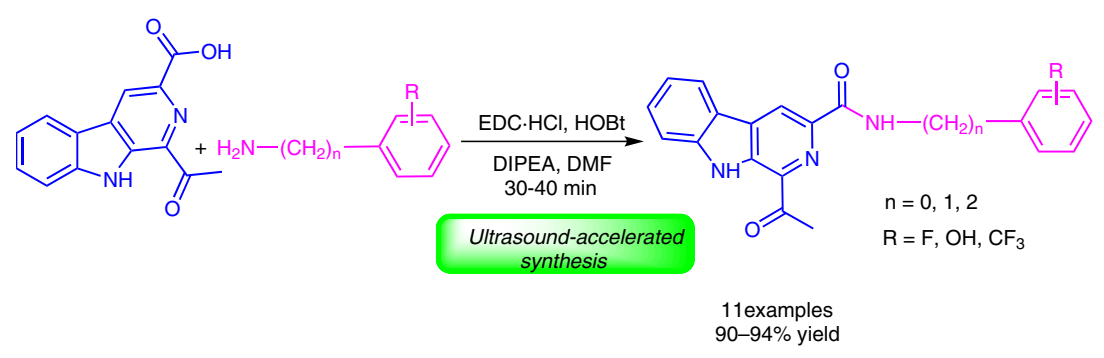

1-Acetyl-3-carboxamide- $\beta$-carboline derivatives have Received: 03.02.2017

Accepted after revision: 12.03 .2017

Published online:

DOI: 10.1055/s-0036-1588167; Art ID: so-2017-d0002-I

License terms: $(C) \bigoplus \$$

Abstract Several biologically important 1 -acetyl-3-carboxamide- $\beta$ carboline derivatives were rapidly synthesized by ultrasound-promoted amide coupling of 1-acetyl-9H-pyrido[3,4-b]indole-3-carboxylic acid with substituted aromatic amines. The major advantages of the proposed method are that use of ultrasound irradiations afforded the desired products in a drastically reduced reaction time and in excellent yields compared with conventional stirring.

Key words ultrasound, $\beta$-carboline, amide coupling, polycyclic indoles

Marine natural products have increasingly become major leads in drug discovery, often showing a unique biochemical mode of action. ${ }^{1,2}$ Indoles continue to attract extensive synthetic interest, due to their divergent pharmacological activities and also because the rigid framework can lead to compounds of marked selectivity in their interactions with enzymes or receptors. ${ }^{3-8}$

The $\beta$-carboline ring system containing a pyridoindole structure is a component of structures with a vast spectrum of biological properties, ${ }^{9-16}$ such as antimicrobial, ${ }^{17}$ antiviral, ${ }^{18}$ antitumor, ${ }^{19,20}$ anticonvulsant, ${ }^{21}$ and parasiticidal activity. ${ }^{22}$ Other $\beta$-carboline derivatives inhibit cyclin-dependent kinase (CDK) 1, IkappaB kinase (IKK), and topoisomerase $I^{23}$ However, an important challenge is the scarce natural availability of marine $\beta$-carbolines, which hinders biological screening in structure-activity relationship (SAR) studies. Therefore, efficient chemical synthesis ${ }^{24}$ of these marine compounds in larger quantities is necessary to investigate their biological activities and is the focus of the work reported herein. been synthesized by a biocatalytic pathway using the McbA enzyme. ${ }^{25}$ However, yields of the target compounds are not high. Additionally, such biocatalytic approaches take longer to establish on an industrial scale. ${ }^{26}$ Other synthetic approaches suffer from drawbacks such as multistep protocols, ${ }^{27}$ or extended reaction times ${ }^{28}$ with overall yields of $19 \%$ and $72 \%$, respectively. Thus, there remains a need for the development of more efficient, convenient and operationally simple approaches for the rapid synthesis of 1 -acetyl-3-carboxamide- $\beta$-carboline derivatives.

Ultrasound-assisted organic reactions have emerged as an innovative technique in a wide variety of conversions. ${ }^{27-}$ ${ }^{30}$ Use of ultrasound irradiation results in accelerated reaction rates, energy conservation and minimization of waste as compared with traditional methods. ${ }^{31}$ In continuation of our interest in the synthesis of a wide range of heterocyclic systems, ${ }^{32}$ we herein report a novel ultrasound-promoted amide coupling for the rapid synthesis of 1-acetyl-3-carboxamide- $\beta$-carboline derivatives in good to excellent yields with a notable reduction in completion time compared with classical methods of amide coupling. ${ }^{33}$

Firstly, synthesis of $\beta$-carboline derivatives 3a-c, which are already known for their antimalarial activity, ${ }^{34}$ was carried out by reacting 1 -acetyl-9H-pyrido[3,4-b]indole-3-carboxylic acid $(\mathbf{1})^{35}$ with the phenylethanamines $\mathbf{2 a} / \mathbf{2} \mathbf{b}$ and indolyl ethanamine $\mathbf{2 c}$ under ultrasonic irradiation (UI) at room temperature (Table 1). As outlined in Table 1, ultrasound irradiation reduced the completion time of the reactions from several hours to minutes and yields were also improved from $81-83 \%$ (under conventional conditions) to 91-92\%. The NMR spectroscopic and mass spectrometric data were in excellent agreement with those reported previously. ${ }^{34}$ 
Table 1 Synthesis of 1-Acetyl-3-carboxamide- $\beta$-carboline Derivatives under Ultrasound Irradiation or Conventional Stirring ${ }^{a}$<smiles>CC(=O)c1nc(C(=O)O)cc2c1[nH]c1ccccc12</smiles>
$2 a / 2 b / 2 c$ $\mathrm{EDC} \cdot \mathrm{HCl}$ $\mathrm{HOBt}$ DIPEA, DMF

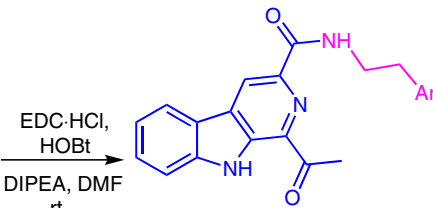

$3 a / 3 b / 3 c$

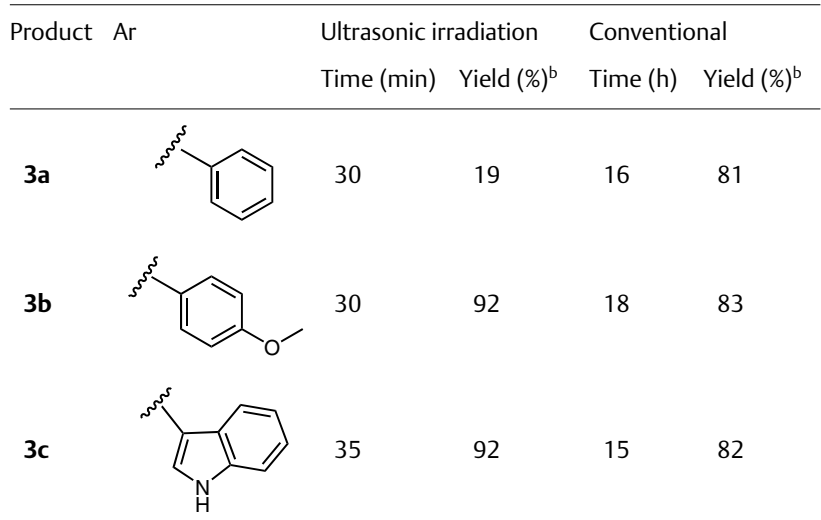

Reaction conditions: 1 (1.0 equiv.), 2a-c (1.2 equiv), DIPEA (2.1 equiv), $\mathrm{EDC} \cdot \mathrm{HCl}$ (1.1 equiv), HOBt (1.1 equiv), DMF, rt.

${ }^{\mathrm{b}}$ Isolated yield.

We extended our study to demonstrate the substrate scope of the reaction with 1 -acetyl-9H-pyrido[3,4-b]indole-3-carboxylic acid (1) using fluorinated and non-fluorinated aromatic amines $\mathbf{2 d - k}$ for the formation of various 1acetyl-3-carboxamide- $\beta$-carboline derivatives $\mathbf{3 d - k}$ in excellent yields of $90-94 \%$ under ultrasonic irradiation (Table 2). All products were analyzed by IR, ${ }^{1} \mathrm{H}$ NMR, ${ }^{13} \mathrm{C}$ NMR and HRMS analysis. From Table 2, it is clear that the reaction accommodated a range of substituents such as fluoro- and trifluoromethyl-groups at different positions on the aromatic ring.

In conclusion, we have reported an ultrasound-accelerated, efficient amide coupling reaction to provide efficient access to 1-acetyl-3-carboxamide- $\beta$-carboline derivatives. The products were obtained in excellent yields with short reaction times and the protocol accommodates a variety of functionality.

\section{Acknowledgment}

The authors are grateful to the SERB, Department of Science \& Technology, for providing financial support and the USIC, University of Delhi for providing instrumentation facilities. NS is grateful to the DST-SERB for a Reseach Associate award and PK to the CSIR for a Senior Research Fellowship.
Table 2 Substrate Scope of the Synthesis of Novel Fluorinated/Nonfluorinated $\beta$-Carboline Derivatives ${ }^{a}$<smiles>CC(=O)c1nc(C(=O)O)cc2c1[nH]c1ccccc12</smiles><smiles>CC(=O)c1nc(C(=O)NCCc2ccc(F)cc2)cc2c1[nH]c1ccccc12</smiles><smiles>CC(=O)c1nc(C(=O)NCc2ccc(F)cc2)cc2c1[nH]c1ccccc12</smiles><smiles>CC(=O)c1nc(C(=O)Nc2ccccc2)cc2c1[nH]c1ccccc12</smiles>

$3 \mathrm{~g}, 90 \%$<smiles>CC(=O)c1nc(C(=O)Nc2cccc(F)c2)cc2c1[nH]c1ccccc12</smiles>

$3 \mathrm{~h}, 93 \%$ $\mathrm{UI}=37 \mathrm{~min}$<smiles>CC(=O)c1nc(C(=O)Nc2ccc(F)cc2F)cc2c1[nH]c1ccccc12</smiles><smiles>CC(=O)c1nc(C(=O)Nc2cc(C(F)(F)F)cc(C(F)(F)F)c2)cc2c1[nH]c1ccccc12</smiles>

a Reaction conditions: 1 ( 1.0 equiv), $\mathbf{2 d}-\mathbf{k}$ (1.2 equiv), DIPEA (2.1 equiv), EDC.HCl (1.1 equiv), HOBt (1.1 equiv), DMF, rt.

${ }^{\mathrm{b}}$ Isolated yield.

\section{Supporting Information}

Supporting information for this article is available online at http://dx.doi.org/10.1055/s-0036-1588167. 


\section{References and Notes}

(1) (a) Blunt, J. W.; Copp, B. R.; Keyzers, R. A.; Munro, M. H. G.; Princep, M. R. Nat. Prod. Rep. 2014, 31, 16. (b) Xiong, Z. Q.; Wang, J. F.; Hao, Y. Y.; Wang, Y. Mar. Drugs 2013, 11, 700.

(2) (a) Cragg, G. M.; Newman, D. J. Biochim. Biophys. Acta 2013, 1830, 3670. (b) Lam, K. S. Curr. Opin. Microbiol. 2006, 9, 245.

(3) Bailey, P. D.; Cochrane, P. J.; Forster, A. H.; Morgana, K. M.; Pearson, D. P. J. Tetrahedron Lett. 1999, 40, 4597.

(4) (a) Zheng, C.; Fang, Y.; Tong, W.; Li, G.; Zhou, H. W.; Lin, Q.; Yang, F.; Yang, Z.; Wang, P.; Peng, Y.; Pang, X.; Yi, Z.; Luo, J.; Liu, M.; Chen, Y. J. Med. Chem. 2014, 57, 600. (b) Winkler, J. D.; Londregan, A. T.; Hamann, M. T. Org. Lett. 2006, 8, 2591.

(5) Tang, J. G.; Wang, Y. H.; Wang, R. R.; Dong, Z. J.; Yang, L. M.; Zheng, Y. T.; Liu, J. K. Chemistry \& Biodiversity 2008, 5, 447.

(6) Kawasaki, T.; Higuchi, K. Nat. Prod. Rep. 2005, 22, 761.

(7) (a) Herraiz, T.; Galisteo, J. J. Agric. Food Chem. 2003, 51, 7156. (b) Chen, H.; Gao, P.; Zhang, M.; Liao, W.; Zhang, J. New J. Chem. 2014, 38, 4155.

(8) Ang, K. K. H.; Holmes, M. J.; Higa, T.; Hamann, M. T.; Kara, U. K. Antimicrob. Agents Chemother. 2000, 44, 1645.

(9) (a) Kusurkar, R. S.; Goswami, S. K.; Vyas, S. M. Tetrahedron Lett. 2003, 44, 4761. (b) Laine, A. E.; Lood, C.; Koskinen, A. M. P. Molecules 2014, 19, 1544.

(10) Airaksinen, M. M.; Kari, I. Med. Biol. 1981, 59, 21.

(11) Carbrera, G. M.; Seldes, A. M. J. Nat. Prod. 1999, 62, 759.

(12) Lippke, K. P.; Schunack, W. G.; Wenning, W.; Mueller, W. E. J. Med. Chem. 1983, 26, 499.

(13) Cain, M.; Weber, R. W.; Guzman, F.; Cook, J. M.; Barker, S. A.; Rice, K. C.; Crawley, J. N.; Paul, S. M.; Skolnick, P. J. Med. Chem. 1982, 25, 1081.

(14) Hagen, T. J.; Skolnick, P.; Cook, J. M. J. Med. Chem. 1987, 30, 750.

(15) Dodd, R. H.; Ouannes, C.; Carvalho, L. P.; Valin, A.; Venault, P.; Chapouthier, G.; Rossier, J.; Potier, P. J. Med. Chem. 1985, 28, 824.

(16) Patel, K.; Gadewar, M.; Tripathi, R.; Prasad, S. K.; Patel, D. K. Asian Pac. J. Trop. Biomed. 2012, 2, 660.

(17) Cao, R.; Peng, W.; Wang, Z.; Zu, A. Curr. Med. Chem. 2007, 14, 479.

(18) Tang, J. G.; Wang, Y. H.; Wang, R. R.; Dong, Z. J.; Yang, L. M.; Zheng, Y. T.; Liu, J. K. Chemistry \& Biodiversity 2008, 5, 447.

(19) Bemis, D. L.; Capedice, J. L.; Gorroochurn, P.; Katz, A. Z.; Buttyan, R. Int. J. Oncol. 2006, 29, 1065.

(20) Cao, R.; Peng, W.; Chen, H.; Ma, Y.; Liu, X.; Hou, X.; Guan, H.; Xu, A. Biochem. Biophys. Res. Commun. 2005, 338, 1557.

(21) Dorey, G.; Dubois, L.; Potier, P.; Dodd, R. H. J. Med. Chem. 1995, 38, 189.

(22) Winkler, J. D.; Londregan, A. T.; Hamann, M. T. Org. Lett. 2006, 8, 2591.

(23) Castro, A. C.; Dang, L. C.; Soucy, F.; Grenier, L.; Mazdiyansi, H.; Hottelet, M.; Parent, L.; Pien, C.; Palombella, V. Adams J. Bioorg. Med. Chem. Lett. 2003, 13, 2419.

(24) Nicolaou, K. C.; Vourloumis, D.; Winssinger, N.; Baran, P. S. Angew. Chem. Int. Ed. 2000, 39, 44.

(25) Ji, C.; Chen, Q.; Li, Q.; Huang, H.; Song, Y.; Maa, J.; Ju, J. Tetrahedron Lett. 2014, 55, 4901

(26) Johannes, T.; Simurdiak, M. R.; Zhao, H. Encyclopedia of Chemical Processing. DOI: 10.1081/E-ECHP-120017565.

(27) Liu, Y. Q.; Li, L. H.; Yang, L.; Li, H. Y. Chem. Pap. 2010, 64, 533.

(28) Meciarova, M.; Polackova, V.; Toma, S. Chem. Pap. 2002, 56, 208.

(29) Meciarova, M.; Toma, S.; Babiak, P. Chem. Pap. 2004, 58, 104.
(30) Tabatabaeian, K.; Mamaghani, M.; Mahmoodi, N. O.; Khorshidi, A. Catal. Commun. 2008, 9, 416.

(31) (a) Kumar, V.; Sharma, A.; Sharma, M.; Sharma, U. K.; Sinha, A. K. Tetrahedron 2007, 63, 9718. (b) Sinha, A. K.; Sharma, A.; Joshi, B. P. Tetrahedron 2007, 63, 960.

(32) (a) Sharma, N.; Chundawat, T. S.; Bhagat, S. Synthesis 2016, 48, 4495. (b) Chundawat, T. S.; Sharma, N.; Kumari, P.; Bhagat, S. Synlett 2016, 27, 404. (c) Chundawat, T. S.; Sharma, N.; Kumari, P.; Bhagat, S. Med. Chem. Res. 2016, 25, 2335.

(33) Conventional method for the synthesis of $\mathbf{9 H}$-pyrido[3,4b]indole-3-carboxamide derivatives (3a-k); General procedure: To a stirred solution of $\mathbf{1}$ (1 equiv) in DMF were added $\mathrm{EDC} \cdot \mathrm{HCl}$ (1.1 equiv) and $\mathrm{HOBt}$ (1.1 equiv), followed by addition of DIPEA (2.1 equiv). The resulting reaction mixture was stirred at r.t. for 30 minutes. The requisite amine $\mathbf{2 a - c}$ was added portionwise and the reaction was stirred at r.t. for 15-18 h (Table 1). Progress of reaction was monitored by TLC. After completion, the reaction mixture was poured into ice-cold water, and the precipitate filtered. Column chromatography on silica (100-200 mesh), eluting with $30-40 \%$ ethyl acetate/hexane gave the pure 1-acetyl-3-carboxamide- $\beta$-carboline derivatives $\mathbf{3 a - c .}$

Ultrasound method for the synthesis of 1-acetyl-3-carboxamide- $\beta$-carboline derivatives (3a-k); General procedure: To a stirred solution of $\mathbf{1}$ (1equiv) in DMF were added EDC. $\mathrm{HCl}$ (1.1 equiv) and HOBt (1.1 equiv), followed by addition of DIPEA (2.1 equiv). The resulting reaction mixture was stirred at r.t. for 10 minutes. The requisite amine $\mathbf{2 a - k}$ was added portionwise and the reaction was stirred at r.t. under sonication for the time detailed in Table 1 and Table 2. The progress of the reaction was monitored by TLC. After completion, the reaction mixture was poured into ice-cold water, and the precipitate filtered. Column chromatography on silica (100-200 mesh), eluting with 30-40\% ethyl acetate/hexane gave the pure 1-acetyl-3-carboxamide- $\beta$ carboline derivatives 3a-k.

\section{Representative Spectroscopic Data}

1-Acetyl- $N$-phenethyl-9H-pyrido[3,4-b]indole-3-carboxamide (3a): Pale-yellow solid; $\mathrm{mp} 172-174{ }^{\circ} \mathrm{C}$; IR (KBr): 3349 , 2914, 1683, $1534 \mathrm{~cm}^{-1} ;{ }^{1} \mathrm{H} \mathrm{NMR}\left(400 \mathrm{MHz}, \mathrm{CDCl}_{3}\right): \delta=10.31(\mathrm{~s}$, $1 \mathrm{H},-N H), 9.00(\mathrm{~s}, 1 \mathrm{H}), 8.12(\mathrm{~d}, J=7.63 \mathrm{~Hz}, 1 \mathrm{H}), 8.00(\mathrm{t}, 1 \mathrm{H}$, $-\mathrm{NH}), 7.56-7.50(\mathrm{~m}, 2 \mathrm{H}), 7.31-7.18(\mathrm{~m}, 6 \mathrm{H}), 3.78(\mathrm{q}, J=6.78 \mathrm{~Hz}$, $2 \mathrm{H}), 2.93(\mathrm{t}, J=6.78 \mathrm{~Hz}, 2 \mathrm{H}), 2.67(\mathrm{~s}, 3 \mathrm{H}) ;{ }^{13} \mathrm{C} \mathrm{NMR}(100 \mathrm{MHz}$, $\left.\mathrm{CDCl}_{3}\right): \delta=202.2,164.4,141.4,139.1,139.0,136.1,133.4,132.5$, 129.6, 128.8, 128.7, 126.5, 122.2, 121.4, 120.9, 118.2, 112.1, 40.4, 35.8, 25.6; HRMS (ESI): $m / z[M+H]^{+}$calcd for $\mathrm{C}_{22} \mathrm{H}_{19} \mathrm{~N}_{3} \mathrm{O}_{2}$ : 358.1555; found: 358.1545 .

1-Acetyl- $N$-(2,4-difluorophenyl)-9H-pyrido[3,4-b]indole-3carboxamide (3j): Yellow solid; mp $210-212{ }^{\circ} \mathrm{C}$; IR (KBr): 3350 , 2916, 1665, $1539 \mathrm{~cm}^{-1} ;{ }^{1} \mathrm{H}$ NMR (400 MHz, DMSO-d $\left.d_{6}\right): \delta=12.29$ $(\mathrm{s}, 1 \mathrm{H},-N H), 10.39(\mathrm{~s}, 1 \mathrm{H},-N H), 9.19(\mathrm{~s}, 1 \mathrm{H}), 8.12(\mathrm{q}$, $J=6.10 \mathrm{~Hz}, 1 \mathrm{H}), 7.83(\mathrm{~d}, J=7.63 \mathrm{~Hz}, 1 \mathrm{H}), 7.63(\mathrm{t}, J=8.39 \mathrm{~Hz}$, $1 \mathrm{H}), 7.46-7.41(\mathrm{~m}, 1 \mathrm{H}), 7.34(\mathrm{t}, J=7.63 \mathrm{~Hz}, 1 \mathrm{H}), 7.17(\mathrm{t}$, $J=8.39 \mathrm{~Hz}, 1 \mathrm{H}), 2.93(\mathrm{~s}, 3 \mathrm{H}) ;{ }^{13} \mathrm{C}$ NMR $\left(100 \mathrm{MHz}, \mathrm{CDCl}_{3}\right): \delta=$ 200.6, 162.5, 145.1, 142.9, 142.4, 137.2, 135.0, 133.9, 133.9, $132.2,129.5,122.4,121.0,120.2,118.4,113.4,111.4,104.5$, 104.2, 25.9; HRMS (ESI): $m / z[M+H]^{+}$calcd for $\mathrm{C}_{20} \mathrm{H}_{13} \mathrm{~F}_{2} \mathrm{~N}_{3} \mathrm{O}_{2}$ : 366.1054; found: 366.1061

(34) Huang, H.; Yao, Y.; He, Z.; Yang, T.; Ma, J.; Tian, X.; Li, Y.; Huang, C.; Chen, X.; Li, W.; Zhang, S.; Zhang, C.; Ju, J. J. Nat. Prod. 2011, $74,2122$.

(35) Tang, J.-G.; Liu, H.; Zhou, Z.-Y.; Liu, J.-K. Synth. Commun. 2010, $40,1411$. 